\title{
Does 3D-Assisted Acetabular Fracture Surgery Improve Surgical Outcome and Physical Functioning?-A Systematic Review
}

\author{
Anne M. L. Meesters ${ }^{1, *(1)}$, Neeltje M. Trouwborst ${ }^{1}$, Jean-Paul P. M. de Vries ${ }^{2}$, Joep Kraeima ${ }^{3}$, Max J. H. Witjes ${ }^{3}$, \\ Job N. Doornberg ${ }^{4}$, Inge H. F. Reininga ${ }^{1} \mathbb{D}$, Frank F. A. IJpma ${ }^{1,+} \mathbb{C}$ and Kaj ten Duis ${ }^{1,+}$ \\ 1 Department of Trauma Surgery, University Medical Center Groningen, University of Groningen, \\ 9713 GZ Groningen, The Netherlands; n.m.trouwborst@umcg.nl (N.M.T.); i.h.f.reininga@umcg.nl (I.H.F.R.); \\ f.f.a.ijpma@umcg.nl (F.F.A.I.); k.ten.duis@umcg.nl (K.t.D.) \\ 2 Department of Surgery, University Medical Center Groningen, University of Groningen, \\ 9713 GZ Groningen, The Netherlands; j.p.p.m.de.vries@umcg.nl \\ 3 3D Lab, Department of Oral and Maxillofacial Surgery, University Medical Center Groningen, \\ University of Groningen, 9713 GZ Groningen, The Netherlands; j.kraeima@umcg.nl (J.K.); \\ m.j.h.witjes@umcg.nl (M.J.H.W.) \\ 4 Department of Orthopaedic Surgery, University Medical Center Groningen, University of Groningen, \\ 9713 GZ Groningen, The Netherlands; j.n.doornberg@umcg.nl \\ * Correspondence: a.m.l.meesters@umcg.nl; Tel.: +31-(0)50-361-61-61 \\ + The last two authors contributed equally to this manuscript.
}

Citation: Meesters, A.M.L.; Trouwborst, N.M.; de Vries, J.-P.P.M.; Kraeima, J.; Witjes, M.J.H.; Doornberg, J.N.; Reininga, I.H.F.; IJpma, F.F.A.; ten Duis, K. Does 3D-Assisted Acetabular Fracture Surgery Improve Surgical Outcome and Physical Functioning?-A Systematic Review. J. Pers. Med. 2021, 11, 966.

https://doi.org/10.3390/jpm11100966

Academic Editor: Monique J. Roobol

Received: 16 July 2021

Accepted: 23 September 2021

Published: 27 September 2021

Publisher's Note: MDPI stays neutral with regard to jurisdictional claims in published maps and institutional affiliations.

Copyright: (c) 2021 by the authors. Licensee MDPI, Basel, Switzerland. This article is an open access article distributed under the terms and conditions of the Creative Commons Attribution (CC BY) license (https:// creativecommons.org/licenses/by/ $4.0 /)$.

\begin{abstract}
Three-dimensional technology is increasingly being used in acetabular fracture treatment. No systematic reviews are available about the added clinical value of 3D-assisted acetabular fracture surgery compared to conventional surgery. Therefore, this study aimed to investigate whether 3Dassisted acetabular fracture surgery compared to conventional surgery improves surgical outcomes in terms of operation time, intraoperative blood loss, intraoperative fluoroscopy usage, complications, and postoperative fracture reduction, and whether it improves physical functioning. Pubmed and Embase databases were searched for articles on 3D technologies in acetabular fracture surgery, published between 2010 and February 2021. The McMaster critical review form was used to assess the methodological quality. Differences between 3D-assisted and conventional surgery were evaluated using the weighted mean and odds ratios. Nineteen studies were included. Three-dimensional-assisted surgery resulted in significantly shorter operation times $(162.5 \pm 79.0$ versus $296.4 \pm 56.0 \mathrm{~min})$, less blood loss $(697.9 \pm 235.7 \mathrm{~mL}$ versus $1097.2 \pm 415.5 \mathrm{~mL})$, and less fluoroscopy usage $(9.3 \pm 5.9$ versus $22.5 \pm 20.4$ times). The odds ratios of complications and fracture reduction were 0.5 and 0.4 for functional outcome in favour of 3D-assisted surgery, respectively. Three-dimensional-assisted surgery reduces operation time, intraoperative blood loss, fluoroscopy usage, and complications. Evidence for the improvement of fracture reduction and functional outcomes is limited.
\end{abstract}

Keywords: acetabular fracture; 3D; three-dimensional; 3D print; surgical planning; systematic review

\section{Introduction}

Acetabular fractures are fractures involving the hip socket, which might have major impacts on the patient's mobility, social activities, and the ability to work. These severe injuries usually occur due to high-energy trauma mechanisms (i.e., car accidents) in young patients [1]. In addition, acetabular fractures are increasingly caused by low-energy trauma mechanisms (i.e., fall at ground level) in frail elderly [1]. Adequate fracture reduction and fixation is crucial to minimise the risks on progressive posttraumatic arthritis of the hip socket and the subsequent need for revision surgery to a total hip arthroplasty [2]. Acetabular fractures are complex fractures, due to the three-dimensional (3D) geometry of the pelvis and displacement of fracture fragments in multiple directions. Insight into fracture patterns can be challenging using only two-dimensional (2D) images [3]. In the past decade, 3D technology has increasingly been used in acetabular fracture treatment. 
Three-dimensional printing is useful for classifying acetabular fractures and for teaching purposes [4-7]. For instance, 3D printed models may improve the quality of surgical trainees' preoperative understanding of the spatial complexity of fractures [8]. In addition, a randomised controlled trial showed that using a 3D interactive software system for teaching acetabular fracture classification improved the classification accuracy [7]. Moreover, the use of 3D printed fracture models has improved fracture classification in comparison with 2D/3D CT images, due to enhanced tactile feedback of the complex geometry $[5,6]$. This may result in a shorter time needed to classify the acetabular fractures and a higher interobserver agreement as compared to the evaluation of these fractures using 2D CT images [4].

Over the past few years, the number of publications on the applications of 3D-assisted surgery in acetabular fracture treatment is rapidly increasing [9]. It encompasses a spectrum of modalities, including 3D visualisation, 3D printing, and patient-specific surgical guides or implants. Preoperative planning of the fracture reduction and pre-contouring of implants using 3D printed models has been reported in acetabular fracture surgery in case series [10-15]. For example, Hu et al. [11] created virtual 3D models of fractured acetabula based on CT images and virtually reduced the fracture fragments, in order to gain more insight into fracture patterns and treatment strategies. Moreover, the uninjured hemipelvis can be mirrored virtually and $3 \mathrm{D}$ printed $[13,15]$. This printed hemipelvis can be used as a template for the pre-contouring of implants prior to surgery $[13,15]$. In addition, the use of $3 \mathrm{D}$ printed drilling guides and patient-specific osteosynthesis plates have been described [16-19]. For instance, 3D printed drilling guides have been designed to fit temporarily on top of an implant in order to aim the drill bit and screw trajectories in the predetermined directions [19]. In addition, patient-specific implants, with or without drilling guides, have been designed based on virtual 3D models $[16,17]$. The application of patient-specific osteosynthesis plates provides the possibility to execute the preoperative plan and attain the predetermined osteosynthesis plate and screw positions [16]. However, comparative studies or reviews on the added clinical value of 3D-assisted acetabular fracture surgery compared to conventional surgery (i.e., defined as using only radiographs and 2D CT images in preoperative planning) are only sparingly available. Next to the surgeons understanding of these technologies, patients cannot be informed properly about the potential benefits of these innovations. In addition, insurance companies take evidence-based decisions on the implementation of these technologies.

Therefore, a systematic review was conducted in order to assess differences in surgical outcome and physical functioning between 3D-assisted and conventional (2D) acetabular fracture treatment. Research questions were: (1) Does 3D-assisted acetabular fracture surgery compared to conventional surgery improve surgical outcomes in terms of operation time, intraoperative blood loss, intraoperative fluoroscopy usage, complications, and postoperative fracture reduction?; and (2) does 3D-assisted acetabular fracture surgery compared to conventional surgery improve physical functioning in terms of Patient-Reported Outcome Measures?

\section{Materials and Methods}

The Preferred Reporting Items for Systematic Reviews (PRISMA) [20] were used. The review protocol has been registered in the PROSPERO International prospective register of systematic reviews under registration number CRD42021225274.

\subsection{Search Strategy and Study Selection}

On 1 March 2021, the PubMed and Embase libraries were searched for articles published between 1 January 2010 and 28 February 2021. Together with a medical librarian, the search string was generated (Table 1). 
Table 1. Search string.

\begin{tabular}{|c|c|}
\hline Database & Search String \\
\hline PubMed & $\begin{array}{l}\text { (3D[tiab] OR three dimension*[tiab] OR } 3 \text { dimension*[tiab] OR ‘Printing, } \\
\text { Three-Dimensional' [Mesh] OR 'Imaging, Three-Dimensional' [Mesh]) } \\
\text { AND (acetabul*[tiab] OR 'Acetabulum' [Mesh]) AND (fractur*[tiab] OR } \\
\text { 'Fractures, Bone' [Mesh]) AND ‘2010/01/01' [PDat]: ‘3000/12/31' [PDat] }\end{array}$ \\
\hline Embase & $\begin{array}{l}\text { ('three dimensional imaging' /exp OR 'three dimensional printing'/ exp OR } \\
\text { '3 d':ti,ab OR ‘3 dimension*':ti,ab OR ‘three dimension*':ti,ab) AND } \\
\text { ('acetabulum' / exp OR acetabul*:ti,ab) AND ('fracture' / exp OR } \\
\text { fractur*:ti,ab) AND [embase]/lim AND } \\
{[2010,2011,2012,2013,2014,2015,2016,2017,2018,2019,2020,2021] / \mathrm{py}}\end{array}$ \\
\hline
\end{tabular}

Studies that were eligible for inclusion were randomised controlled trials, cohort studies, case-control studies, cross-sectional studies, and case series on the treatment of acetabular fractures in humans by using 3D technology. Exclusion criteria were reviews; letters to the editor or conference abstracts; cadaveric studies; case reports $(\mathrm{N}<10)$; paediatric studies (age < 18 years); studies in other languages than English, German, French, or Dutch; studies on fracture classification, measurements or education; studies on intraoperative imaging or surgical navigation; and biomechanical studies. Articles were imported into Rayyan QCRI, a web-based sorting tool for systematic literature reviews [21]. Next, two reviewers (AM, FIJ) independently screened the articles for eligibility based on the titles and abstracts using the Rayyan QCRI tool. The same reviewers independently screened all remaining articles by full text. Finally, the references of the included articles were screened for additional relevant manuscripts.

\subsection{Quality Check and Data Extraction}

The guidelines of the McMaster University Occupational Therapy Evidence-Based Practice Research Group were used to assess the methodological quality and risk of bias [22]. The McMaster critical appraisal consists of components considering the study purpose, background literature, study design, sample size, randomisation, outcome measures, study intervention, study results, conclusions, and implications. Scores were given with 'yes $=1$ point', 'no $=0$ points', and 'not applicable (NA)'. The total score reflects the methodological quality with a maximum score of 16 for RCTs, 12 for case series, and 14 for other designs. The definitive score is presented as a percentage that varies from 0 to $100 \%$, with a higher score indicating a higher methodological quality. Scores of $<50 \%$ are considered poor-quality studies, scores of $50-74 \%$ are considered moderate-quality studies, scores of $75-90 \%$ are considered good-quality studies, and scores of $>90 \%$ are considered excellent-quality studies. The data extraction and quality check were independently conducted (AM, FIJ) using the McMaster Critical Review Form. Disagreements were resolved in a consensus meeting.

\subsection{Outcome Measures}

The primary outcome of this systematic review was the surgical outcome in terms of operation time, intraoperative blood loss, intraoperative fluoroscopy usage, complications, and fracture reduction. Complications were defined as nerve injury, vascular injury, infection, thrombosis/embolism, heterotopic ossification, osteoarthritis, avascular necrosis of the femoral head, and implant failure. The quality of acetabular fracture reduction was defined by the greatest residual gap or step-off at the acetabulum on the plain radiographs or on a postoperative CT scan in any of the views $[23,24]$. The residual displacement was graded according to Matta's criteria as anatomic (0 to $1 \mathrm{~mm}$ gap and/or step-off), imperfect ( 2 to $3 \mathrm{~mm}$ ), or poor $(>3 \mathrm{~mm}$ ) [24]. An adequate reduction was defined as the Matta category anatomical and satisfactory or a postoperative displacement of $\leq 2 \mathrm{~mm}$, and a poor reduction was defined as the Matta category poor or a postoperative displacement of $>2 \mathrm{~mm}$. Secondary outcome was physical functioning, assessed with the Patient-Reported 
Outcome Measures (PROMs) or clinician-reported outcome measures. Functional outcome was graded according to the definitions of the Modified Merle d'Aubigné (Excellent 18, Good 15-17, Fair 13-14, Poor < 13) and the Harris Hip score (Excellent 90-100, Good 80-90, Fair 70-80, Poor <70) [25-28].

\subsection{Statistical Analysis}

The weighted mean with a standard deviation of all applicable studies was calculated, using SPSS (version 23, IBM, Chicago, IL, USA), when more than two studies reported the outcome variable. For comparative studies, the differences in continuous outcome measures were calculated by using the inverse variance weighting method and presented as the weighted mean difference (WMD) with the $95 \%$ confidence interval (CI), using Review Manager (version 5.4.1, The Nordic Cochrane Centre, The Cochrane Collaboration, Copenhagen, Denmark). For dichotomous variables, the odds ratio with the $95 \%$ CI was calculated using the Mantel-Haenszel method in Review Manager. A $p$-value of $<0.05$ was considered to indicate statistical significance. Authors were contacted to retrieve additional data, such as not reported means or their standard deviations, but retrieving additional data was unsuccessful.

\section{Results}

\subsection{Search and Study Characteristics}

In total, 482 studies were found. After removal of duplicates, 357 studies were screened on title and abstract. After title and abstract screening, 28 articles were included for full-text screening. Nine of these full-text articles were excluded due to the following reasons: foreign language article on 3D printing and pre-contouring the implant $(\mathrm{N}=1)$; case reports $(\mathrm{N}=2)$; descriptive study $(\mathrm{N}=1)$; biomechanical study $(\mathrm{N}=1)$; conference abstract $(\mathrm{N}=1)$; outcome measurements unclear $(\mathrm{N}=3)$. In total, 19 studies met the inclusion criteria for this systematic review (Figure 1) [29-47]. The included studies enrolled a total of 753 patients (median sample size 27; range 10-146). Three-dimensional-assisted surgery was used in 478 of all the patients (Figure 2). In 400 patients, a 3D print and plate precontouring of the implant was used (14 studies); in 69 patients, a patient-specific implant was used (three studies); and in 9 patients, only 3D printing for pre- and intraoperative fracture visualisation was used (one study). Conventional surgery, defined as preoperative planning based on radiographs and 2DCT images (axial, sagittal, and coronal views), was used in 275 patients. The study characteristics are presented in Table 2.

\subsection{Methodological Quality Assessment}

Three randomised controlled trials $[38,43,44]$, one prospective cohort study [40], ten case control studies [30-32,34,35,41,42,45-47], and five case series [29,33,36,37] were included. The methodological quality of the papers varied from low (Table 3) to good (Table 4). The median and interquartile range (IQR) McMaster score was 69\% (IQR 64-86) for all studies together and for the prospective and retrospective studies separately. 


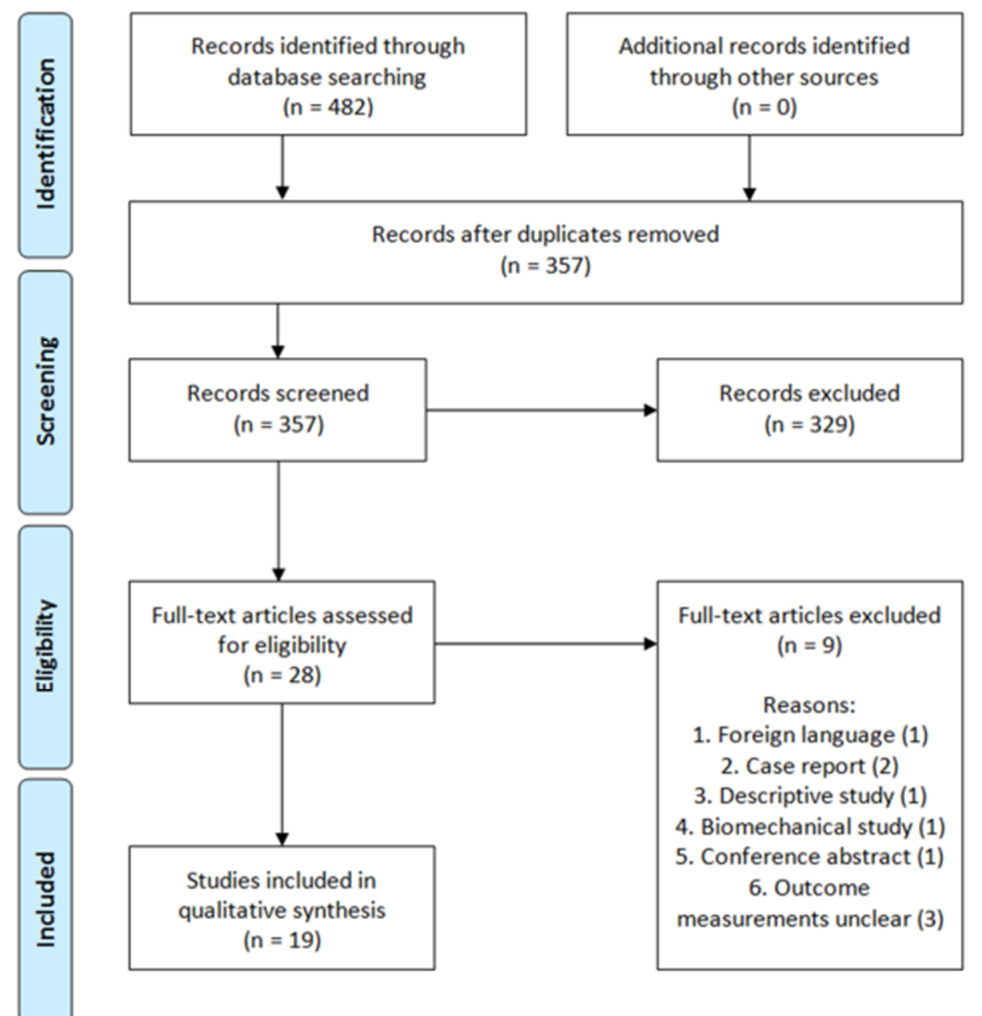

Figure 1. PRISMA flow diagram.

Table 2. Study characteristics.

\begin{tabular}{|c|c|c|c|c|c|c|c|}
\hline Study & Year & Country & Design & $\mathbf{N}$ & Period & Outcome Measurements & 3D Technology \\
\hline $\begin{array}{l}\text { Ansari } \\
\text { et al. [30] }\end{array}$ & 2020 & India & Case control & 27 & $\begin{array}{c}\text { August 2017-July } \\
2018\end{array}$ & $\begin{array}{l}\text { Operation time, intraoperative blood loss, } \\
\text { intraoperative fluoroscopy usage, } \\
\text { postoperative fracture reduction, } \\
\text { complications, FU: Harris hip score }\end{array}$ & $\begin{array}{l}\text { 3D printing and plate } \\
\text { pre-contouring }\end{array}$ \\
\hline $\begin{array}{l}\text { Chen et al. } \\
\text { [31] }\end{array}$ & 2019 & China & Case control & 52 & $\begin{array}{c}\text { January } \\
\text { 2013-January } 2017\end{array}$ & $\begin{array}{c}\text { Operation time, intraoperative blood loss, } \\
\text { postoperative fracture reduction, } \\
\text { complications, FU: modified Merle } \\
\text { d'Aubigné }\end{array}$ & $\begin{array}{l}\text { 3D printing and plate } \\
\text { pre-contouring; } \\
\text { virtual plating }\end{array}$ \\
\hline $\begin{array}{l}\text { Downey } \\
\text { et al. [40] }\end{array}$ & 2020 & Ireland & $\begin{array}{l}\text { Prospective } \\
\text { cohort }\end{array}$ & 18 & $\begin{array}{c}\text { October 2017-May } \\
2018\end{array}$ & $\begin{array}{l}\text { Operation time, intraoperative blood loss, } \\
\text { intraoperative fluoroscopy usage, } \\
\text { postoperative fracture reduction, } \\
\text { complications: infection }\end{array}$ & 3D printing \\
\hline $\begin{array}{l}\text { Hsu et al. } \\
\text { [41] }\end{array}$ & 2019 & China & Case control & 29 & $\begin{array}{l}\text { March } \\
\text { 2014-February } \\
2018\end{array}$ & $\begin{array}{l}\text { Operation time, intraoperative blood loss, } \\
\text { postoperative fracture reduction, } \\
\text { complications }\end{array}$ & $\begin{array}{l}\text { 3D printing and plate } \\
\text { pre-contouring }\end{array}$ \\
\hline $\begin{array}{l}\text { Huang } \\
\text { et al. [38] }\end{array}$ & 2020 & China & $\begin{array}{l}\text { Randomised } \\
\text { Controlled } \\
\text { Trial }\end{array}$ & 40 & $\begin{array}{l}\text { September } \\
\text { 2013-September } \\
2017\end{array}$ & $\begin{array}{l}\text { Operation time, intraoperative blood loss, } \\
\text { intraoperative fluoroscopy usage, } \\
\text { postoperative fracture reduction, } \\
\text { complications, FU: Harris hip score }\end{array}$ & $\begin{array}{l}\text { 3D printing and plate } \\
\text { pre-contouring }\end{array}$ \\
\hline $\begin{array}{l}\text { IJpma et al. } \\
\text { [39] }\end{array}$ & 2021 & Netherlands & $\begin{array}{l}\text { Prospective } \\
\text { case series }\end{array}$ & 10 & $\begin{array}{l}\text { January } \\
\text { 2017-December } \\
2018 \\
\end{array}$ & $\begin{array}{c}\text { Postoperative fracture reduction, } \\
\text { complications, FU: Short Musculoskeletal } \\
\text { Function Assessment }\end{array}$ & $\begin{array}{l}\text { Patient-specific } \\
\text { implants }\end{array}$ \\
\hline $\begin{array}{l}\text { Li et al. } \\
\text { [42] }\end{array}$ & 2019 & Taiwan & Case control & 16 & $\begin{array}{c}\text { September } \\
\text { 2013-August } 2017\end{array}$ & $\begin{array}{l}\text { Operation time, intraoperative blood loss, } \\
\text { postoperative fracture reduction, } \\
\text { complications }\end{array}$ & $\begin{array}{l}\text { 3D printing and plate } \\
\text { pre-contouring }\end{array}$ \\
\hline $\begin{array}{l}\text { Maini et al. } \\
\text { [43] }\end{array}$ & 2018 & India & $\begin{array}{l}\text { Randomised } \\
\text { Controlled } \\
\text { Trial }\end{array}$ & 21 & $\begin{array}{l}\text { June } \\
\text { 2012-December } \\
2014\end{array}$ & $\begin{array}{l}\text { Operation time, intraoperative blood loss, } \\
\text { postoperative fracture reduction, } \\
\text { complications }\end{array}$ & $\begin{array}{l}\text { 3D printing and plate } \\
\text { pre-contouring }\end{array}$ \\
\hline
\end{tabular}


Table 2. Cont.

\begin{tabular}{|c|c|c|c|c|c|c|c|}
\hline Study & Year & Country & Design & $\mathbf{N}$ & Period & Outcome Measurements & 3D Technology \\
\hline $\begin{array}{l}\text { Maini et al. } \\
\text { [44] }\end{array}$ & 2018 & India & $\begin{array}{l}\text { Randomised } \\
\text { Controlled } \\
\text { Trial }\end{array}$ & 25 & $\begin{array}{l}\text { October } \\
\text { 2014-March } 2016\end{array}$ & $\begin{array}{l}\text { Operation time, intraoperative blood loss, } \\
\text { postoperative fracture reduction }\end{array}$ & $\begin{array}{l}\text { 3D printing of } \\
\text { virtually } \\
\text { pre-contoured plates } \\
\text { as template for plate } \\
\text { pre-contouring }\end{array}$ \\
\hline $\begin{array}{l}\text { Öztürk } \\
\text { et al. [45] }\end{array}$ & 2020 & Turkey & Case control & 18 & $\begin{array}{l}\text { January 2017-June } \\
2018\end{array}$ & $\begin{array}{l}\text { Operation time, intraoperative blood loss, } \\
\text { intraoperative fluoroscopy usage, } \\
\text { postoperative fracture reduction, } \\
\text { complications }\end{array}$ & $\begin{array}{l}3 \mathrm{D} \text { printing and plate } \\
\text { pre-contouring }\end{array}$ \\
\hline $\begin{array}{l}\text { Wan et al. } \\
\text { [46] }\end{array}$ & 2019 & China & Case control & 96 & $\begin{array}{l}\text { January 2016-June } \\
2017\end{array}$ & $\begin{array}{l}\text { Operation time, intraoperative blood loss, } \\
\text { intraoperative fluoroscopy usage, } \\
\text { postoperative fracture reduction, } \\
\text { complications, FU: Harris hip score }\end{array}$ & $\begin{array}{l}3 \mathrm{D} \text { printing and plate } \\
\text { pre-contouring }\end{array}$ \\
\hline $\begin{array}{l}\text { Wang et al. } \\
\text { [47] }\end{array}$ & 2020 & China & Case control & 50 & $\begin{array}{l}\text { January 2016-June } \\
2017\end{array}$ & $\begin{array}{c}\text { Operation time, intraoperative blood loss, } \\
\text { postoperative fracture reduction, } \\
\text { complications }\end{array}$ & $\begin{array}{l}\text { Patient-specific } \\
\text { implants }\end{array}$ \\
\hline $\begin{array}{l}\text { Wang et al. } \\
\text { [32] }\end{array}$ & 2020 & China & Case control & 88 & $\begin{array}{l}\text { February } \\
\text { 2013-February } \\
2016\end{array}$ & $\begin{array}{l}\text { Operation time, intraoperative blood loss, } \\
\text { postoperative fracture reduction, } \\
\text { complications, FU: Merle d'Aubigne }\end{array}$ & $\begin{array}{l}\text { 3D printing and plate } \\
\text { pre-contouring }\end{array}$ \\
\hline $\begin{array}{l}\text { Weidert } \\
\text { et al. [33] }\end{array}$ & 2020 & Germany & $\begin{array}{l}\text { Retrospective } \\
\text { case series }\end{array}$ & 12 & NS & $\begin{array}{c}\text { Operation time, intraoperative blood loss, } \\
\text { FU: (modified) Harris hip score, Merle } \\
\text { d'Aubigne }\end{array}$ & $\begin{array}{l}\text { 3D printing and plate } \\
\text { pre-contouring }\end{array}$ \\
\hline $\begin{array}{l}\text { Wu et al. } \\
\text { [34] }\end{array}$ & 2020 & China & Case control & 43 & $\begin{array}{l}\text { May 2014-January } \\
2018\end{array}$ & $\begin{array}{c}\text { Operation time, intraoperative blood loss, } \\
\text { postoperative fracture reduction, } \\
\text { complications, FU: modified Merle } \\
\text { d'Aubigne }\end{array}$ & $\begin{array}{l}\text { Patient-specific } \\
\text { implants }\end{array}$ \\
\hline $\begin{array}{l}\text { Xu et al. } \\
\text { [29] }\end{array}$ & 2014 & China & $\begin{array}{l}\text { Prospective } \\
\text { case series }\end{array}$ & 24 & $\begin{array}{c}\text { January } \\
\text { 2008-August } 2011\end{array}$ & $\begin{array}{l}\text { Operation time, intraoperative blood loss, } \\
\text { postoperative fracture reduction, FU: } \\
\text { Merle d'Aubigne, complications }\end{array}$ & $\begin{array}{l}\text { Patient-specific } \\
\text { implants }\end{array}$ \\
\hline $\begin{array}{l}\text { Yu et al. } \\
\text { [35] }\end{array}$ & 2020 & China & Case control & 146 & $\begin{array}{l}\text { June } \\
\text { 2011-December } \\
2017\end{array}$ & $\begin{array}{l}\text { Operation time, intraoperative blood loss, } \\
\text { intraoperative fluoroscopy usage, } \\
\text { postoperative fracture reduction, } \\
\text { complications, FU: Harris hip score }\end{array}$ & $\begin{array}{l}\text { 3D printing and plate } \\
\text { pre-contouring }\end{array}$ \\
\hline $\begin{array}{l}\text { Zeng et al. } \\
\text { [36] }\end{array}$ & 2016 & China & $\begin{array}{l}\text { Prospective } \\
\text { case series }\end{array}$ & 10 & $\begin{array}{l}\text { June } \\
\text { 2013-February } \\
2015\end{array}$ & $\begin{array}{l}\text { Postoperative fracture reduction, } \\
\text { complications }\end{array}$ & $\begin{array}{l}\text { 3D printing and plate } \\
\text { pre-contouring }\end{array}$ \\
\hline $\begin{array}{l}\text { Zou et al. } \\
\text { [37] }\end{array}$ & 2020 & China & $\begin{array}{l}\text { Retrospective } \\
\text { case series }\end{array}$ & 33 & $\begin{array}{l}\text { June } \\
\text { 2017-December } \\
2018\end{array}$ & $\begin{array}{c}\text { Operation time, intraoperative blood loss, } \\
\text { postoperative fracture reduction, } \\
\text { complications, FU: modified Merle } \\
\text { d'Aubigne }\end{array}$ & $\begin{array}{l}\text { 3D printing and plate } \\
\text { pre-contouring }\end{array}$ \\
\hline
\end{tabular}




\section{D printing process:}

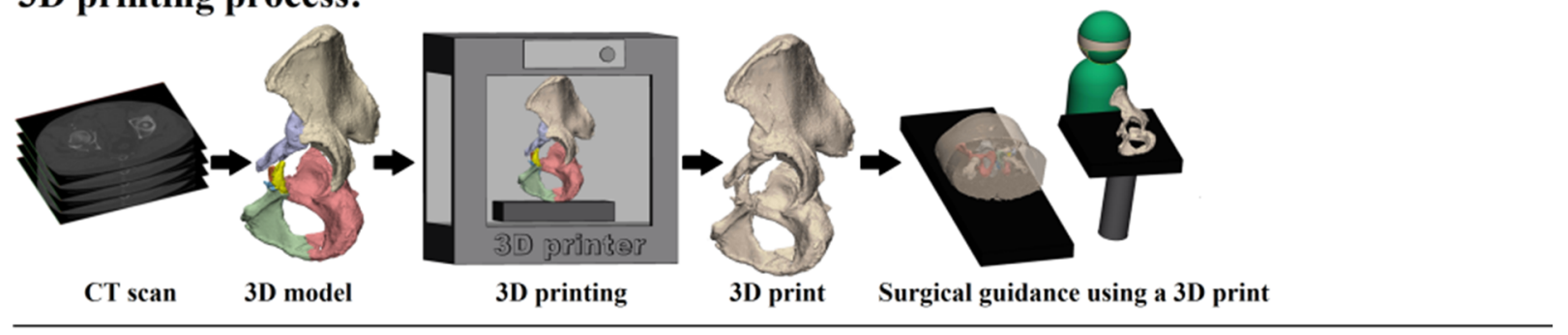

\section{D printing and pre-contouring of the implant:}

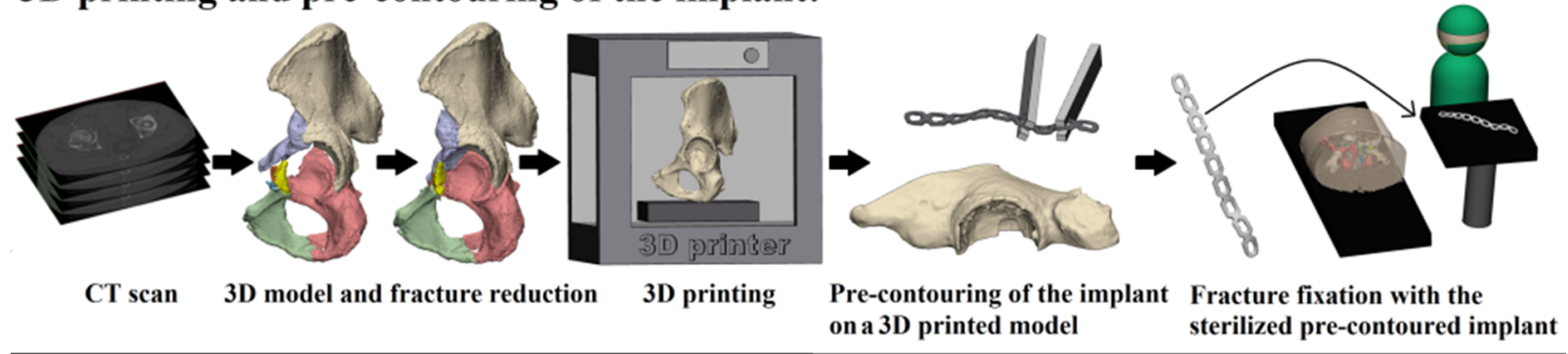

\section{Patient-specific implants:}

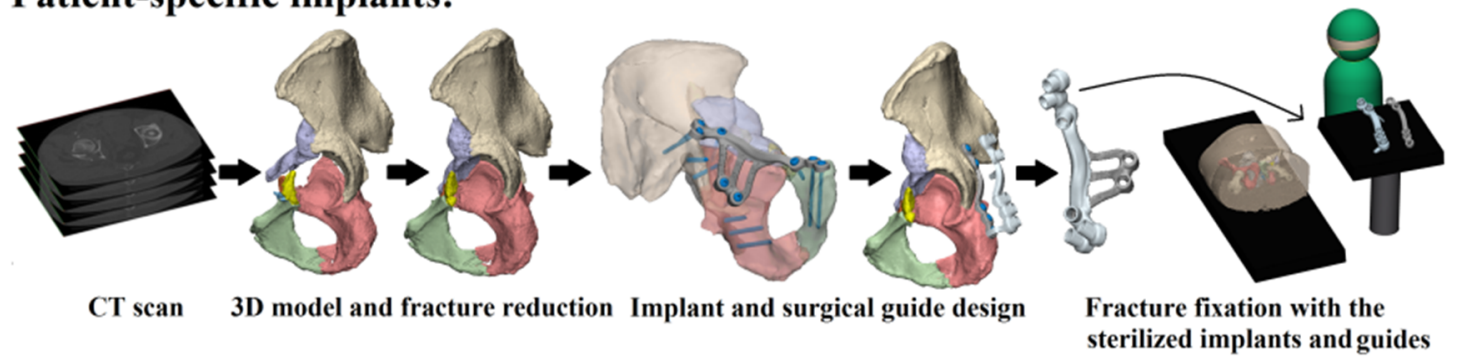

Figure 2. Three-dimensional-assisted surgery. Three-dimensional-assisted surgery encompasses a spectrum of modalities, including 3D visualisation, 3D printing, and patient-specific surgical guides or implants. The steps required for 3D printing, 3D printing and pre-contouring of the implant, or the manufacturing of patient-specific implants are illustrated. In the 3D printing process (top row) a virtual 3D model is created from a CT scan, e.g., using Mimics Medical software in which a threshold for bone tissue is selected based on the Hounsfield Units of the CT scan. The 3D models are split into the separate fragments, indicated by the different colours. This virtual model can be 3D printed and used for preoperative planning and surgical guidance. For 3D printing and pre-contouring of the implant (middle row), a virtual 3D model is created from a CT scan. Then, the contralateral healthy hemipelvis is mirrored, e.g., using 3-matic Medical software, and it is used as a template for the virtual fracture reduction. The fracture fragments are virtually reduced to their original anatomical position. The mirrored or virtually reduced hemipelvis can be 3D printed and this 3D print is used for pre-contouring of the implant. One study performed virtual plating and printed the contour of a plate, which was then used for pre-contouring the implant [44]. Next, the pre-contoured implant is sterilised and used for intraoperative fracture fixation. Finally, patient-specific implants (bottom row) are designed, based on the virtual 3D model from the CT scan. Either the mirrored contralateral pelvis or the fracture reduction can be used as a model for the implants. The screw directions and positions are predetermined and then the implant is designed based on the shape of the pelvis of the individual patient and based on the fracture type. The implant is accompanied by a surgical guide, to ensure that the screws are positioned and directed as planned. The implants and surgical guides are sterilised and used for intraoperative fracture fixation within four days. 
Table 3. Quality assessment part one.

\begin{tabular}{|c|c|c|c|c|c|c|c|c|c|}
\hline Categories & $\begin{array}{l}\text { Zou, } \\
2020\end{array}$ & $\begin{array}{l}\text { Weidert, } \\
2020\end{array}$ & $\begin{array}{l}\text { Zeng, } \\
2016\end{array}$ & $\begin{array}{l}\text { Öztürk, } \\
2020\end{array}$ & $\begin{array}{l}\text { Wan, } \\
2019\end{array}$ & $\begin{array}{c}\mathrm{Xu}, \\
2014\end{array}$ & $\begin{array}{l}\text { Maini, } \\
2018^{1}\end{array}$ & $\begin{array}{c}\mathrm{Li}, \\
2019\end{array}$ & $\begin{array}{l}\text { Wang, } \\
2020^{2}\end{array}$ \\
\hline \multicolumn{10}{|l|}{ 1. Study purpose } \\
\hline Was the study question clearly stated? & 0 & 0 & 0 & 1 & 0 & 1 & 1 & 1 & 0 \\
\hline \multicolumn{10}{|l|}{ 2. Literature review } \\
\hline $\begin{array}{l}\text { Was relevant background literature } \\
\text { reviewed? }\end{array}$ & 0 & 0 & 1 & 1 & 0 & 0 & 1 & 1 & 1 \\
\hline 3. Study design & $\mathrm{CR}$ & $\mathrm{CR}$ & $\mathrm{CR}$ & $\mathrm{CC}$ & $\mathrm{CC}$ & $\mathrm{CR}$ & RCT & $\mathrm{CC}$ & $\mathrm{CC}$ \\
\hline \multicolumn{10}{|l|}{ 4. Sample } \\
\hline Was the sample described in detail? & 1 & 0 & 1 & 1 & 1 & 1 & 1 & 1 & 1 \\
\hline Was the sample justified? & 0 & 0 & 0 & 0 & 0 & 0 & 0 & 0 & 0 \\
\hline Were the groups randomised? & 0 & 0 & 0 & 0 & 0 & 0 & 1 & 0 & 0 \\
\hline Was randomising appropriate done? & NA & NA & NA & NA & NA & NA & 1 & NA & NA \\
\hline \multicolumn{10}{|l|}{ 5. Outcomes } \\
\hline Were the outcome measures reliable? & 0 & 1 & 0 & 0 & 1 & 1 & 1 & 0 & 1 \\
\hline Were the outcome measures valid? & 0 & 1 & 1 & 0 & 1 & 1 & 1 & 0 & 1 \\
\hline \multicolumn{10}{|l|}{ 6. Intervention } \\
\hline Intervention was described in detail? & 1 & 1 & 1 & 1 & 0 & 1 & 1 & 1 & 1 \\
\hline Contamination was avoided? & NA & NA & NA & 1 & 1 & 1 & 1 & 1 & 1 \\
\hline Cointervention was avoided? & NA & NA & NA & 1 & 1 & 0 & 1 & 1 & 1 \\
\hline \multicolumn{10}{|l|}{ 7. Results } \\
\hline $\begin{array}{l}\text { Results were reported in terms of } \\
\text { statistical significance? }\end{array}$ & 0 & 0 & 0 & 1 & 1 & 0 & 0 & 1 & 1 \\
\hline $\begin{array}{l}\text { Were the analysis method/s } \\
\text { appropriate? }\end{array}$ & 0 & 0 & 0 & 0 & 1 & 0 & 0 & 1 & 1 \\
\hline Clinical importance was reported? & 1 & 1 & 1 & 1 & 1 & 1 & 1 & 1 & 1 \\
\hline Drop-outs were reported? & 0 & 0 & 0 & 0 & 0 & 0 & 0 & 0 & 0 \\
\hline \multicolumn{10}{|l|}{ 8. Conclusion } \\
\hline $\begin{array}{l}\text { Conclusions were appropriate given } \\
\text { study methods and results? }\end{array}$ & 0 & 1 & 0 & 0 & 0 & 1 & 0 & 1 & 0 \\
\hline Total & $3 / 12$ & $5 / 12$ & $5 / 12$ & $8 / 14$ & $8 / 14$ & $7 / 12$ & $11 / 16$ & $10 / 14$ & $10 / 14$ \\
\hline$\%$ & 25 & 42 & 42 & 57 & 57 & 58 & 69 & 71 & 71 \\
\hline
\end{tabular}

Yes $=1$ point, no $=0$ points, $C C=$ Case Control study, $\mathrm{RCT}=$ Randomised Controlled Trial, $\mathrm{CR}=$ Case Series, N/A = Not applicable. ${ }^{1}$ : Maini et al. (2018) -Evaluation of accuracy of virtual surgical planning for patient-specific pre-contoured plate in acetabular fracture fixation. ${ }^{2}$ : Wang et al. (2020)-The effect of new preoperative preparation method compared to conventional method in complex acetabular fractures: minimum 2-year follow-up. 
Table 4. Quality assessment part two.

\begin{tabular}{|c|c|c|c|c|c|c|c|c|c|c|}
\hline Categories & $\begin{array}{l}\text { Huang, } \\
2020\end{array}$ & $\begin{array}{l}\text { Maini, } \\
2018^{3}\end{array}$ & $\begin{array}{r}\text { Wu, } \\
2020\end{array}$ & $\begin{array}{l}\text { IJpma, } \\
2021\end{array}$ & $\begin{array}{c}\text { Ansari, } \\
2020\end{array}$ & $\begin{array}{l}\text { Chen, } \\
2019\end{array}$ & $\begin{array}{l}\text { Downey, } \\
2020\end{array}$ & $\begin{array}{l}\text { Hsu, } \\
2019\end{array}$ & $\begin{array}{l}\text { Wang, } \\
2020^{4}\end{array}$ & $\begin{array}{c}\text { Yu, } \\
2020\end{array}$ \\
\hline \multicolumn{11}{|l|}{ 1. Study purpose } \\
\hline $\begin{array}{l}\text { Was the study question clearly } \\
\text { stated? }\end{array}$ & 0 & 1 & 1 & 1 & 1 & 1 & 1 & 1 & 1 & 1 \\
\hline \multicolumn{11}{|l|}{ 2. Literature review } \\
\hline $\begin{array}{l}\text { Was relevant background literature } \\
\text { reviewed? }\end{array}$ & 1 & 1 & 0 & 1 & 1 & 1 & 1 & 1 & 1 & 0 \\
\hline 3. Study design & $\mathrm{RCT}$ & $\mathrm{RCT}$ & $\mathrm{CC}$ & CS & $\mathrm{CC}$ & $\mathrm{CC}$ & CS & $\mathrm{CC}$ & $\mathrm{CC}$ & $\mathrm{CC}$ \\
\hline \multicolumn{11}{|l|}{ 4. Sample } \\
\hline Was the sample described in detail? & 1 & 1 & 1 & 1 & 1 & 1 & 1 & 1 & 1 & 1 \\
\hline Was the sample justified? & 0 & 0 & 0 & 0 & 0 & 0 & 0 & 0 & 0 & 0 \\
\hline Were the groups randomised? & 1 & 1 & 0 & 0 & 0 & 0 & 0 & 0 & 0 & 0 \\
\hline $\begin{array}{l}\text { Was randomising appropriate } \\
\text { done? }\end{array}$ & 0 & 1 & NA & NA & NA & NA & NA & NA & NA & NA \\
\hline \multicolumn{11}{|l|}{ 5. Outcomes } \\
\hline $\begin{array}{l}\text { Were the outcome measures } \\
\text { reliable? }\end{array}$ & 1 & 1 & 1 & 1 & 1 & 1 & 1 & 1 & 1 & 1 \\
\hline Were the outcome measures valid? & 1 & 0 & 1 & 1 & 1 & 1 & 1 & 1 & 1 & 1 \\
\hline \multicolumn{11}{|l|}{ 6. Intervention } \\
\hline $\begin{array}{l}\text { Intervention was described in } \\
\text { detail? }\end{array}$ & 1 & 1 & 1 & 1 & 1 & 1 & 1 & 1 & 1 & 1 \\
\hline Contamination was avoided? & 1 & 1 & 1 & NA & 1 & 1 & 1 & 1 & 1 & 1 \\
\hline Cointervention was avoided? & 1 & 1 & 1 & NA & 1 & 1 & 1 & 1 & 1 & 1 \\
\hline \multicolumn{11}{|l|}{ 7. Results } \\
\hline $\begin{array}{l}\text { Results were reported in terms of } \\
\text { statistical significance? }\end{array}$ & 1 & 1 & 1 & 1 & 1 & 1 & 1 & 1 & 1 & 1 \\
\hline $\begin{array}{l}\text { Were the analysis method/s } \\
\text { appropriate? }\end{array}$ & 1 & 1 & 1 & 1 & 1 & 1 & 1 & 1 & 1 & 1 \\
\hline Clinical importance was reported? & 1 & 1 & 1 & 1 & 1 & 1 & 1 & 1 & 1 & 1 \\
\hline Drop-outs were reported? & 0 & 0 & 0 & 0 & 0 & 0 & 0 & 0 & 0 & 1 \\
\hline \multicolumn{11}{|l|}{ 8. Conclusion } \\
\hline $\begin{array}{l}\text { Conclusions were appropriate } \\
\text { given study methods and results? }\end{array}$ & 1 & 0 & 1 & 1 & 1 & 1 & 1 & 1 & 1 & 1 \\
\hline Total & $12 / 16$ & $12 / 16$ & $11 / 14$ & $10 / 12$ & $12 / 14$ & $12 / 14$ & $12 / 14$ & $12 / 14$ & $12 / 14$ & $12 / 14$ \\
\hline$\%$ & 75 & 75 & 79 & 83 & 86 & 86 & 86 & 86 & 86 & 86 \\
\hline
\end{tabular}

Yes = 1 point, no = 0 points, CC = Case Control study, CS = Cohort study, RCT = Randomised Controlled Trial, CR = Case Series, N/A = Not applicable. ${ }^{3}$ : Maini et al. (2018) - Three-dimensional printing and patient-specific pre-contoured plate: future of acetabulum fracture fixation? ${ }^{4}$ : Wang et al. (2020)—Three-dimensional printing of patient-specific plates for the treatment of acetabular fractures involving quadrilateral plate disruption.

\subsection{Surgical Outcomes}

The weighted mean operation time in the 3D-assisted group and in the conventional group was $162.5 \pm 79.0 \mathrm{~min}$ versus $296.4 \pm 56.0 \mathrm{~min}$. Additionally, the weighted mean blood loss of all studies was $697.9 \pm 235.7 \mathrm{~mL}$ versus $1097.2 \pm 415.5 \mathrm{~mL}$. Nine out of fourteen comparative studies reported a significantly shorter operation time and less blood loss when 3D-assisted surgery was performed [30-32,34,38,41,45-47]. The operation time was 43 min shorter for the 3D-assisted group compared to the conventional group, but the 
heterogeneity was high (Figure 3). There was $243 \mathrm{~mL}$ less blood loss in the 3D-assisted group compared to the conventional group, but the heterogeneity was high (Figure 4).

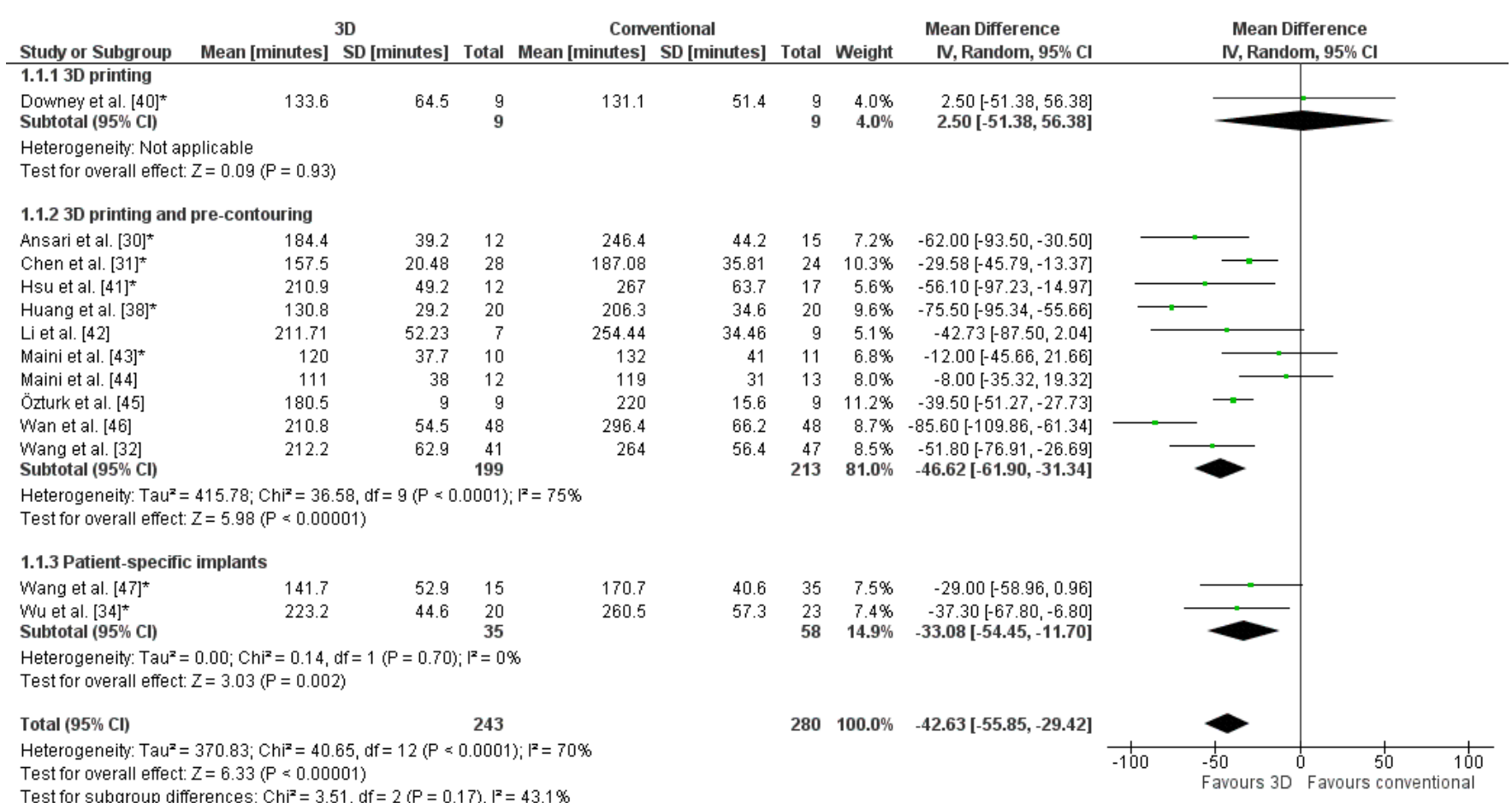

Figure 3. Forest plot of operation time. *: Good-quality study.

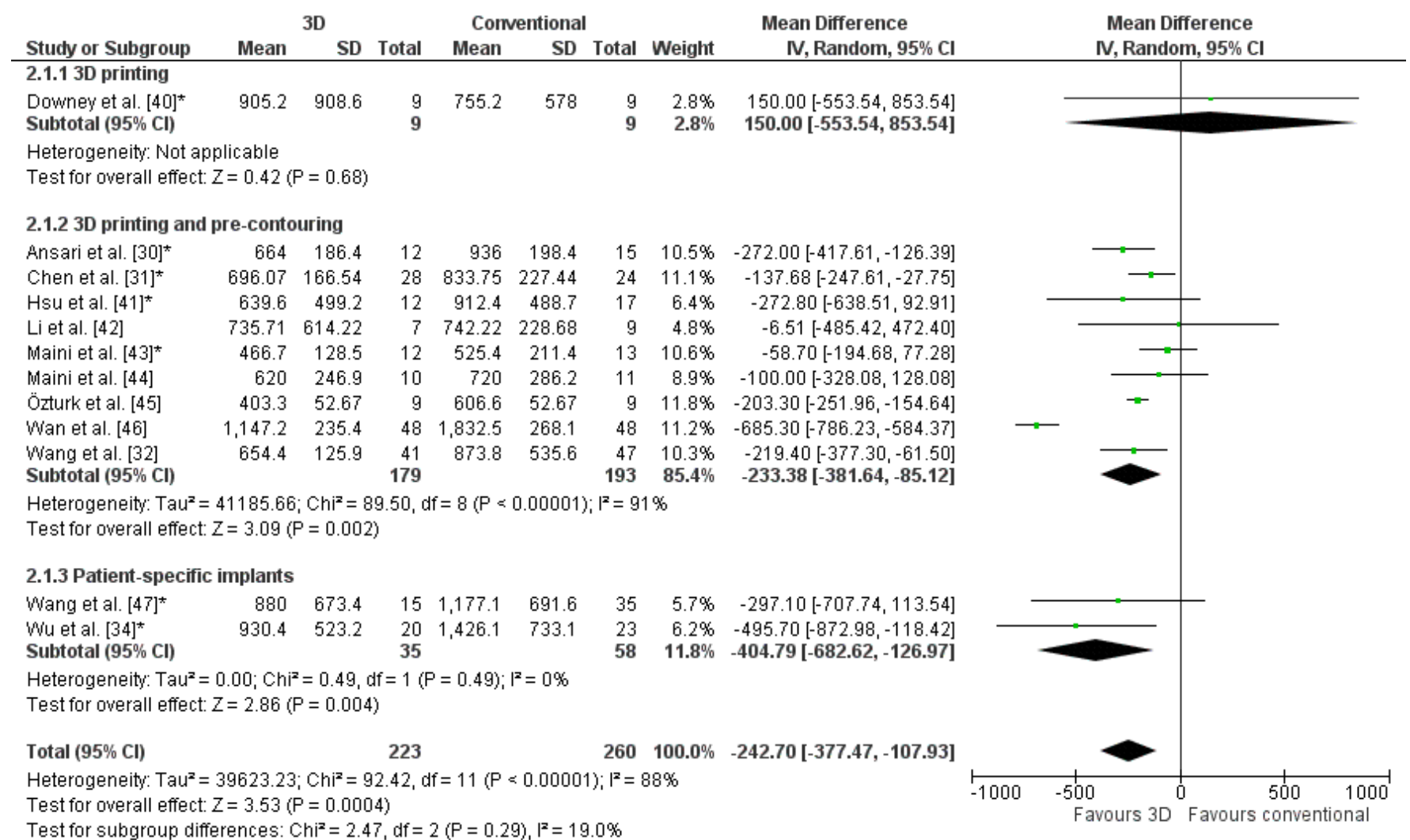

Figure 4. Forest plot of blood loss. *: Good-quality study. 
The weighted mean of three studies reporting on fluoroscopy frequency was $9.3 \pm 5.9$ times in the 3D-assisted group and $22.5 \pm 20.4$ times in the conventional group [30,45,46]. Additionally, one study using 3D prints reported a fluoroscopy dose of $1078.1 \pm 800.3 \mathrm{mGycm}^{2}$ in the 3D-assisted group and $727.1 \pm 349.4 \mathrm{mGycm}^{2}$ in the conventional group [40]. In addition, one study using $3 \mathrm{D}$ prints and pre-contouring of implants reported a significant decrease $(p<0.001)$ in fluoroscopy time in the 3D-assisted group $(4.2 \pm 1.8 \mathrm{~s})$ compared to the conventional group $(7.7 \pm 2.6 \mathrm{~s})$ [38].

The odds ratio for complications was significantly lower for 3D-assisted surgery (OR: 0.5, Figure 5). Two studies reported no complications in both groups $[40,45]$. For the comparative studies using 3D printing and pre-contouring of the implants, 41 out of 187 patients $(22 \%)$ had a complication in the 3D-assisted group, compared to 70 out of 200 patients (35\%) in the conventional group. In the two comparative studies using patientspecific implants, four complications (11\%) occurred in the 3D-assisted group, compared to ten complications (19\%) in the conventional group.

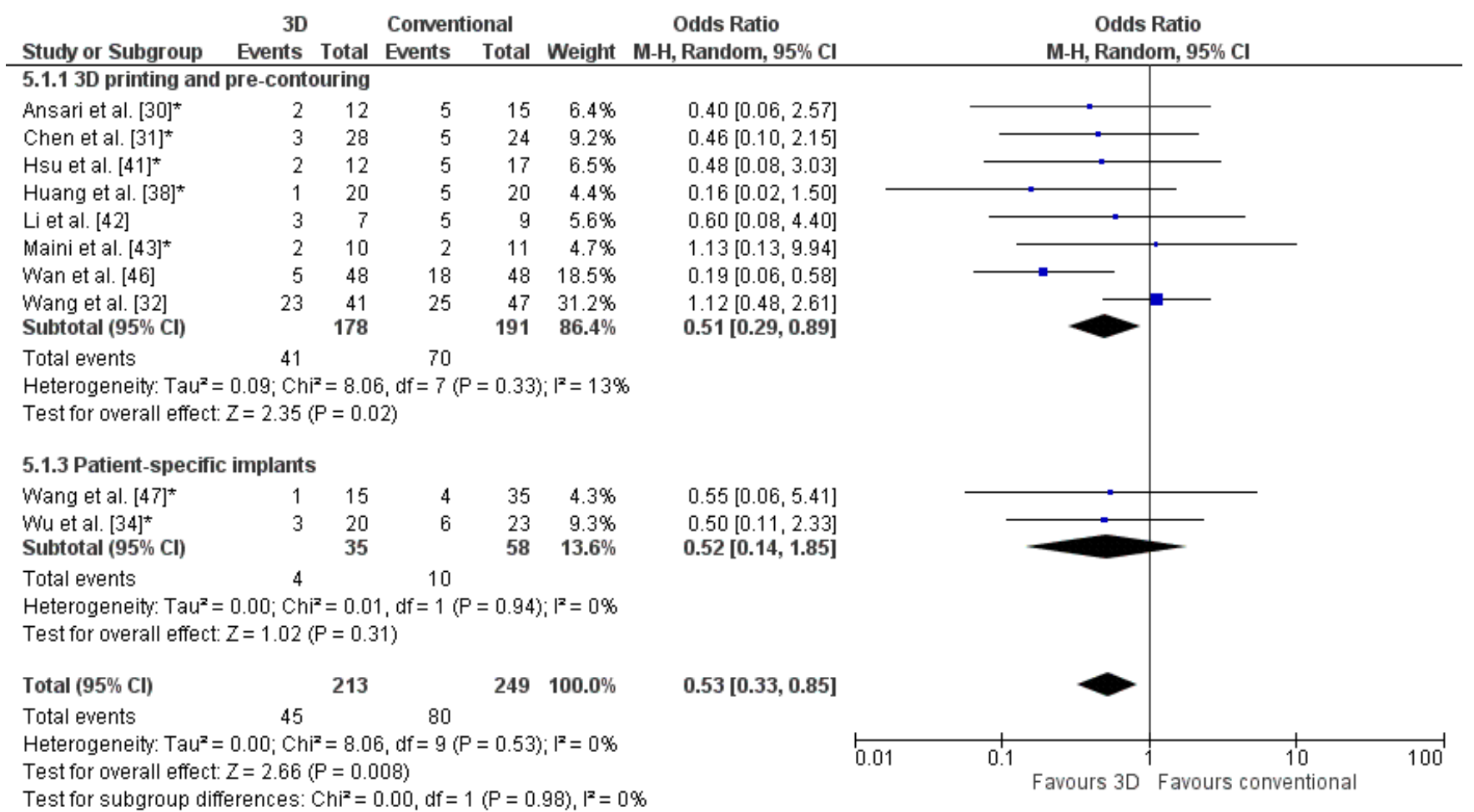

Figure 5. Forest plot of the complications. *: Good-quality study.

The weighted mean of the residual fracture displacement was $3.1 \pm 1.4$ (range 2-5) $\mathrm{mm}$ for the 3D-assisted group and $3.7 \pm 2.0$ (range 2-8) $\mathrm{mm}$ for the conventional group. The odds ratio of a poor reduction was significantly lower for 3D-assisted surgery (OR: 0.5, Figure 6). Two studies reported a better reduction in the 3D-assisted group compared to the conventional group ( $p=0.001$ and $p=0.003$ ) [38,47]. In the 3D-assisted group, $14 \%$ of the patients had a poor reduction compared to $24 \%$ in the conventional group.

\subsection{Functional Outcome}

Studies that reported on functional outcome used the Harris Hip or Merle d'Aubigné scores. One study reported a Harris Hip score of $79.7 \pm 13.7$ in the 3D-assisted group and $83.4 \pm 12.3$ in the conventional group [30]. Another study reported a Modified Merle d'Aubigné score of $16.25 \pm 1.64$ for the 3D-assisted group and $15.83 \pm 1.88$ for the conventional group [31]. A significantly lower odds ratio for poor functional outcome was found for 3D-assisted surgery (OR: 0.4, Figure 7). In these studies, $84 \%$ of the patients had a good clinical outcome in the 3D-assisted group, compared to $71 \%$ in the conventional group. 


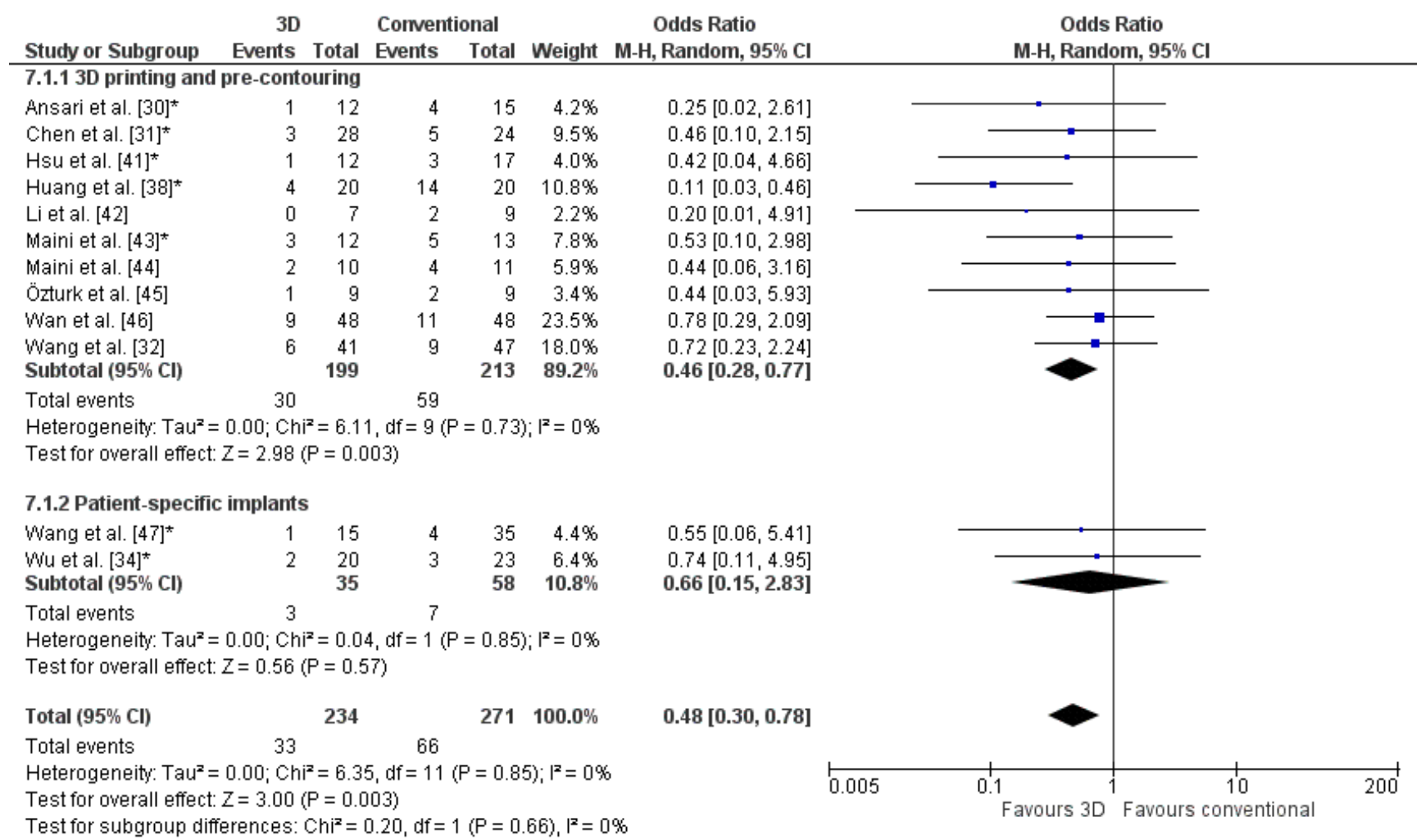

Figure 6. Forest plot of the postoperative reduction, where the events indicate a poor reduction. *: Good-quality study.

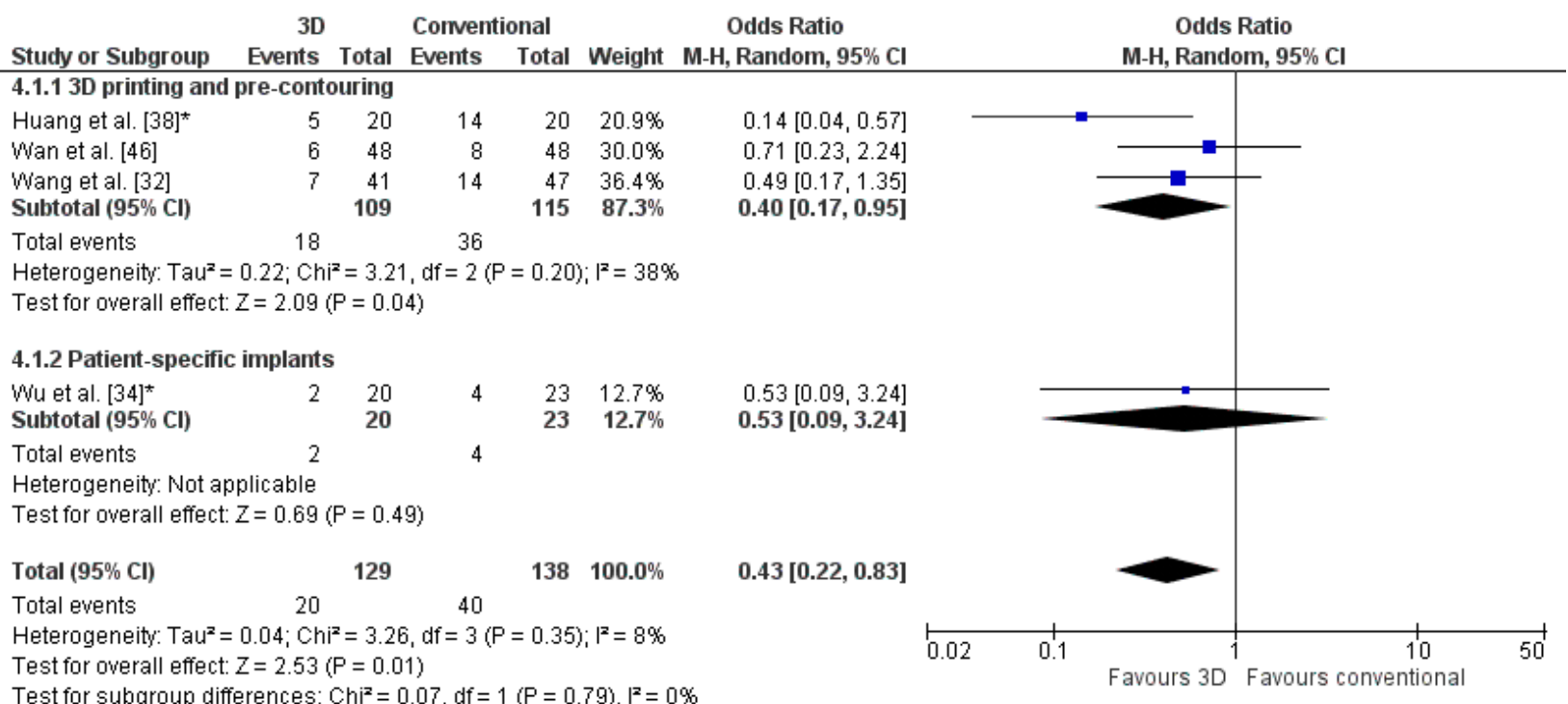

Figure 7. Forest plot of the functional outcome, where the events indicate a poor functional outcome. The Harris Hip score was used by Huang et al. [38] and Wan et al. [46]. The Merle d'Aubigné score was used by Wang et al. [32] and Wu et al. [34].

*: Good-quality study.

\section{Discussion}

Three-dimensional-assisted surgery encompasses a spectrum of modalities, including $3 \mathrm{D}$ visualisation, 3D printing, and patient-specific implants, which can be implemented in the pre- and perioperative phases in acetabular fracture surgery. The added clinical value of 3D-assisted acetabular fracture surgery compared to conventional surgery is still under debate. Therefore, the aim of this systematic review was to assess whether 3Dassisted surgery improves the surgical outcome and physical functioning. Nineteen articles, using either 3D printing, 3D printing and pre-contouring of the implant, or custom-made 
patient-specific implants, were included in this systematic review. The results indicate a positive effect of 3D-assisted surgery on operation time, blood loss, fluoroscopy usage, and complications. Evidence of the improvement in physical functioning and fracture reduction is limited.

Shorter operation time, less intraoperative blood loss, and reduced intraoperative fluoroscopy usage in the 3D-assisted group could be explained by a more efficient surgery due to meticulous preoperative planning. Three-dimensional fracture visualisation and 3D printing give more insight into the fracture characteristics [48]. In addition, the use of 3D technology allows for the planning of screw and implant positions and to subsequently discuss it with seniors prior to the surgery. Due to optimised preparation, screw or implant malposition might be avoided. Moreover, the use of pre-contoured or patient-specific implants might contribute to efficiency as well, because time-consuming intra-operative bending and fitting manoeuvres are no longer necessary [39]. Finally, the quality of the fracture reduction is an important predictor for long-term native hip survivorship [2]. Verbeek et al. [2] found that 3\% of patients with an anatomic reduction (0-1 mm of residual displacement) on CT had conversion to THA compared with $14 \%$ with an imperfect reduction $(2-3 \mathrm{~mm})$, and $36 \%$ with a poor reduction $(>3 \mathrm{~mm})$. In this review, small differences in fracture reduction were found between 3D-assisted and conventional surgery $(3.1 \pm 1.4 \mathrm{~mm}$ versus $3.7 \pm 2.0 \mathrm{~mm})$. Our results were difficult to compare with other large cohort studies due to differences between studies regarding imaging modalities, measurement methods, and reduction criteria [49,50]. The hypothesis was that the positive effect on fracture reduction in the 3D-assisted group could be attributed to preoperative planning of the reduction strategy and an optimal fit of the pre-contoured or patient-specific implants, which possibly serves as a reference for the fracture reduction. Larger trials are needed to assess the effect of 3D-assisted surgery on fracture reduction.

In this systematic review, six comparative studies reported on the functional outcome [30-32,34,38,46]. Overall, little difference in functional outcome was found after $3 \mathrm{D}$-assisted versus conventional surgery ( $84 \%$ versus $71 \%$ good functional outcome). Some studies used the Harris Hip score $[30,38,46]$, whereas others used the Merle d'Aubigné score $[31,32,34]$. Both instruments are, however, not designed and validated for evaluating the functional outcome of acetabular fractures [51]. Comparing results between studies is difficult due to the limited number of studies reporting on the functional outcome and usage of different measurement methods. Three-dimensional-assisted surgery has advantages, but it also takes effort to implement in the workflow. Additional time is needed for preoperative planning. The manufacturing of a 3D print of part of the pelvis for pre-contouring of the implant takes about six to eight hours for printing. This process is often performed and optimised by a team of technical physicians and engineers with expertise of the 3D software and hardware. Software can be either freely available online or CE-certified for medical use, with varying accompanying costs. A simple 3D printer can be used for producing in-hospital nonsterile 3D prints. However, for producing a 3D print for sterile use, one needs a medically certified 3D printer that is more expensive and often operated by an external party. Therefore, future studies about the cost-effectiveness of the 3D technologies are probably needed before they can be used on a large scale.

Limitations of this systematic review include a number of low-quality studies, small patient groups, and the heterogeneity of study populations. Moreover, possible publication bias exists because most studies solely reported positive effects of 3D-assisted surgery. However, all studies were physician-initiated and no studies were sponsored by the industry. More extensive preparation for surgery may contribute to the positive effects on the surgical and functional outcomes in addition to the 3D technology itself. A limitation of using fracture reduction as an outcome measure is that the inter- and intra-observer variabilities of the gap and step-off measurements are high [52]. Moreover, some studies assessed fracture reduction on X-rays, whereas other studies used CT scans, making it difficult to compare and interpret results and causing heterogeneity. 


\section{Conclusions}

The techniques currently used in 3D-assisted acetabular fracture surgery are 3D printing and visual surgical planning, 3D printing and pre-contouring of implants, and custom-made patient-specific implants. Three-dimensional-assisted surgery compared to conventional surgery reduces operation time, intraoperative blood loss, intraoperative fluoroscopy usage, and complication rate. Evidence for the improvement of postoperative fracture reduction and physical functioning is limited, because of heterogeneity and varying qualities of the studies.

Author Contributions: Conceptualization, A.M.L.M., N.M.T. and F.F.A.I.; Methodology, A.M.L.M., N.M.T., K.t.D., I.H.F.R. and F.F.A.I.; Software, A.M.L.M.; Validation, A.M.L.M., N.M.T., J.-P.P.M.d.V., J.K., M.J.H.W., J.N.D., I.H.F.R., F.F.A.I. and K.t.D.; Formal Analysis, A.M.L.M., N.M.T., J.-P.P.M.d.V., J.K., M.J.H.W., J.N.D., I.H.F.R., F.F.A.I. and K.t.D.; Investigation, A.M.L.M. and F.F.A.I.; Resources, A.M.L.M., N.M.T. and F.F.A.I.; Data Curation, A.M.L.M.; Writing-Original Draft Preparation, A.M.L.M., N.M.T., K.t.D. and F.F.A.I.; Writing—Review and Editing, J.P.P.M.d.V., J.K., M.J.H.W., J.N.D. and I.H.F.R.; Visualization, A.M.L.M.; Supervision, J.-P.P.M.d.V., J.K., M.J.H.W., J.N.D., I.H.F.R., F.F.A.I. and K.t.D.; Project Administration, A.M.L.M.; Funding Acquisition, N/A. All authors have read and agreed to the published version of the manuscript.

Funding: This research received no external funding.

Institutional Review Board Statement: Not applicable.

Informed Consent Statement: Not applicable.

Data Availability Statement: The authors declare that the data supporting the findings of this study are available within the paper.

Acknowledgments: We thank the Central Medical Library of the University Medical Center Groningen for their support setting up the search strategy.

Conflicts of Interest: The authors declare no conflict of interest.

\section{References}

1. Rinne, P.P.; Laitinen, M.K.; Huttunen, T.; Kannus, P.; Mattila, V.M. The incidence and trauma mechanisms of acetabular fractures: A nationwide study in Finland between 1997 and 2014. Injury 2017, 48, 2157-2161. [CrossRef]

2. Verbeek, D.O.; van der List, J.P.; Tissue, C.M.; Helfet, D.L. Predictors for Long-Term Hip Survivorship Following Acetabular Fracture Surgery-Importance of Gap Compared with Step Displacement. J. Bone Jt. Surg. 2018, 100, 922-929. [CrossRef] [PubMed]

3. Scheinfeld, M.H.; Dym, A.A.; Spektor, M.; Avery, L.L.; Dym, R.J.; Amanatullah, D.F. Acetabular fractures: What radiologists should know and how 3D CT can aid classification. RadioGraphics 2015, 35, 555-577. [CrossRef] [PubMed]

4. Brouwers, L.; Pull ter Gunne, A.F.; de Jongh, M.A.C.; van der Heijden, F.H.W.M.; Leenen, L.P.H.; Spanjersberg, W.R.; van Helden, S.H.; Verbeek, D.O.; Bemelman, M.; Lansink, K.W.W. The value of 3D printed models in understanding acetabular fractures. 3D Print. Addit. Manuf. 2018, 5, 37-46. [CrossRef]

5. Awan, O.A.; Sheth, M.; Sullivan, I.; Hussain, J.; Jonnalagadda, P.; Ling, S.; Ali, S. Efficacy of 3D Printed Models on Resident Learning and Understanding of Common Acetabular Fracturers. Acad. Radiol. 2019, 26, 130-135. [CrossRef] [PubMed]

6. Manganaro, M.S.; Morag, Y.; Weadock, W.J.; Yablon, C.M.; Gaetke-Udager, K.; Stein, E.B. Creating Three-dimensional Printed Models of Acetabular Fractures for Use as Educational Tools. Radiogr. Rev. Publ. Radiol. Soc. N. Am. Inc. 2017, 37, 871-880. [CrossRef]

7. Wang, H.; Lyu, F.; Sugand, K.; Wong, S.; Lin, Y.; Wang, Q. Learning Acetabular Fracture Classification using a Three-Dimensional Interactive Software: A Randomized Controlled Trial. Anat. Sci. Educ. 2018, 9, 1-9. [CrossRef]

8. Zheng, Y.X.; Yu, D.F.; Zhao, J.G.; Wu, Y.L.; Zheng, B. 3D Printout Models vs. 3D-Rendered Images: Which Is Better for Preoperative Planning? J. Surg. Educ. 2016, 73, 518-523. [CrossRef]

9. Tack, P.; Victor, J.; Gemmel, P.; Annemans, L. 3D-printing techniques in a medical setting: A systematic literature review. Biomed. Eng. Online 2016, 15, 1-22. [CrossRef]

10. Citak, M.; Gardner, M.J.; Kendoff, D.; Tarte, S.; Krettek, C.; Nolte, L.P.; Hüfner, T. Virtual 3D planning of acetabular fracture reduction. J. Orthop. Res. 2008, 26, 547-552. [CrossRef]

11. Hu, Y.; Li, H.; Qiao, G.; Liu, H.; Ji, A.; Ye, F. Computer-assisted virtual surgical procedure for acetabular fractures based on real CT data. Injury 2011, 42, 1121-1124. [CrossRef]

12. Fornaro, J.; Keel, M.; Harders, M.; Marincek, B.; Szekely, G.; Frauenfelder, T. An interactive surgical planning tool for acetabular fractures: Initial results. J. Orthop. Surg. Res. 2010, 5, 50. [CrossRef] 
13. Upex, P.; Jouffroy, P.; Riouallon, G. Application of 3D printing for treating fractures of both columns of the acetabulum: Benefit of pre-contouring plates on the mirrored healthy pelvis. Orthop. Traumatol. Surg. Res. 2017, 103, 331-334. [CrossRef]

14. Yu, A.W.; Duncan, J.M.; Daurka, J.S.; Lewis, A.; Cobb, J. A feasibility study into the sse of three-dimensional printer modelling in acetabular fracture surgery. Adv. Orthop. 2015, 2015, 1-4. [CrossRef]

15. Chana-Rodríguez, F.; Mananes, R.P.; Rojo-Manaute, J.; Gil, P.; Martínez-Gómiz, J.M.; Vaquero-Martín, J. 3D surgical printing and pre contoured plates for acetabular fractures. Injury 2016, 47, 2507-2511. [CrossRef]

16. Merema, B.J.; Kraeima, J.; ten Duis, K.; Wendt, K.W.; Warta, R.; Vos, E.; Schepers, R.H.; Witjes, M.J.H.; IJpma, F.F.A. The design, production and clinical application of 3D patient-specific implants with drilling guides for acetabular surgery. Injury 2017, 48 , 2540-2547. [CrossRef]

17. Wang, D.; Wang, Y.; Wu, S.; Lin, H.; Yang, Y.; Fan, S.; Gu, C.; Wang, J.; Song, C. Customized a Ti6Al4V bone plate for complex pelvic fracture by selective laser melting. Materials 2017, 10, 35. [CrossRef] [PubMed]

18. Brown, G.A.; Milner, B.; Firoozbakhsh, K. Application of computer-generated stereolithography and interpositioning template in acetabular fractures: A report of eight cases. J. Orthop. Trauma 2002, 16, 347-352. [CrossRef] [PubMed]

19. Meesters, A.M.L.; Assink, N.; Ten Duis, K.; Fennema, E.M.; Kraeima, J.; Witjes, M.J.H.; de Vries, J.P.P.M.; Stirler, V.M.A.; Ijpma, F.F.A. Accuracy of patient-specific drilling guides in acetabular fracture surgery: A human cadaver study. J. Pers. Med. 2021, 11, 763. [CrossRef] [PubMed]

20. Moher, D.; Liberati, A.; Tetzlaff, J.; Altman, D.G.; Altman, D.; Antes, G.; Atkins, D.; Barbour, V.; Barrowman, N.; Berlin, J.A.; et al Preferred reporting items for systematic reviews and meta-analyses: The PRISMA statement. PLoS Med. $2009,8,336-341$.

21. Ouzzani, M.; Hammady, H.; Fedorowicz, Z.; Elmagarmid, A. Rayyan-A web and mobile app for systematic reviews. Syst. Rev. 2016, 5, 1-10. [CrossRef]

22. Law, M.; Stewart, D.; Pollock, N.; Letts, L.; Bosch, J.; Westmoreland, M. Guidelines for Critical Review form-Quantitative Studies. 1998. Available online: https:/ / healthsci.mcmaster.ca/docs/librariesprovider130/default-document-library/guidelines-forcritical-review-form-quantiative-studies-english.pdf?sfvrsn=ee9f6c19_2 (accessed on 1 March 2021).

23. Verbeek, D.O.; van der List, J.P.; Moloney, G.B.; Wellman, D.S.; Helfet, D.L. Assessing postoperative reduction following acetabular fracture surgery: A standardized digital CT-based method. J. Orthop. Trauma 2018, 32, e284-e288. [CrossRef] [PubMed]

24. Matta, J.M. Fractures of the acetabulum: Accuracy of reduction and clinical results in patients managed operatively within three weeks after the injury. J. Bone Jt. Surg. 1996, 78, 1632-1645. [CrossRef] [PubMed]

25. Banaszkiewicz, P.A. Traumatic Arthritis of the Hip after Dislocation and Acetabular Fractures: Treatment by Mold Arthroplasty: An End-Result Study Using a New Method of Result Evaluation. In Classic Papers in Orthopaedics, 1st ed.; Springer: London, UK, 2014; pp. 13-17. ISBN 9781447154518.

26. Banaszkiewicz, P.A. Functional Results of Hip Arthroplasty with Acrylic Prosthesis. In Classic Papers in Orthopaedics, 1st ed.; Springer: London, UK, 2014; pp. 19-22. ISBN 9781447154518.

27. Harris, W. Traumatic Arthritis of the Hip after Dislocation and Acetabular Fractures: Treatment by Mold Arthroplasty: An End-Result Study Using a New Method of Result Evaluation. J. Bone Jt. Surg. 1969, 51, 737-755. [CrossRef]

28. D'Aubigne, R.; Postel, M. Functional Results of Hip Arthroplasty with Acrylic Prosthesis. J. Bone Jt. Surg. 1954, 36, 451-475. [CrossRef]

29. Xu, M.; Zhang, L.-H.; Zhang, Y.-Z.; Zhang, L.-C.; He, C.-Q.; Wang, Y.; Tang, P.-F. Custom-made locked plating for acetabular fracture: A pilot study in 24 consecutive cases. Orthopedics 2014, 37, e660-e670. [CrossRef] [PubMed]

30. Ansari, S.; Barik, S.; Singh, S.K.; Sarkar, B.; Goyal, T.; Kalia, R.B. Role of 3D printing in the management of complex acetabular fractures: A comparative study. Eur. J. Trauma Emerg. Surg. 2020. [CrossRef]

31. Chen, K.; Yang, F.; Yao, S.; Xiong, Z.; Sun, T.; Zhu, F.; Telemacque, D.; Drepaul, D.; Ren, Z.; Guo, X. Application of computerassisted virtual surgical procedures and three-dimensional printing of patient-specific pre-contoured plates in bicolumnar acetabular fracture fixation. Orthop. Traumatol. Surg. Res. 2019, 105, 877-884. [CrossRef]

32. Wang, P.; Kandemir, U.; Zhang, B.; Fei, C.; Zhuang, Y.; Zhang, K. The effect of new preoperative preparation method compared to conventional method in complex acetabular fractures: Minimum 2-year follow-up. Arch. Orthop. Trauma Surg. 2020, 141, 215-222. [CrossRef]

33. Weidert, S.; Andress, S.; Linhart, C.; Suero, E.M.; Greiner, A.; Böcker, W.; Kammerlander, C.; Becker, C.A. 3D printing method for next-day acetabular fracture surgery using a surface filtering pipeline: Feasibility and 1-year clinical results. Int. J. Comput. Assist. Radiol. Surg. 2020, 15, 565-575. [CrossRef]

34. Wu, H.-Y.; Shao, Q.-P.; Song, C.-J.; Shang, R.-R.; Liu, X.-M.; Cai, X.-H. Personalized Three-Dimensional Printed Anterior Titanium Plate to Treat Double-Column Acetabular Fractures: A Retrospective Case-Control Study. Orthop. Surg. 2020, 12, $1212-1222$. [CrossRef]

35. Yu, C.; Yu, W.; Mao, S.; Zhang, P.; Zhang, X.; Zeng, X.; Han, G. Traditional three-dimensional printing technology versus three-dimensional printing mirror model technology in the treatment of isolated acetabular fractures: A retrospective analysis. $J$. Int. Med. Res. 2020, 48, 0300060520924250. [CrossRef]

36. Zeng, C.; Xing, W.; Wu, Z.; Huang, H.; Huang, W. A combination of three-dimensional printing and computer-assisted virtual surgical procedure for preoperative planning of acetabular fracture reduction. Injury 2016, 47, 2223-2227. [CrossRef] [PubMed]

37. Zou, R.; Wu, M.; Guan, J.; Xiao, Y.; Chen, X. Therapeutic Effect of Acetabular Fractures Using the Pararectus Approach Combined with 3D Printing Technique. Orthop. Surg. 2020, 12, 1854-1858. [CrossRef] [PubMed] 
38. Huang, J.-H.; Liao, H.; Tan, X.-Y.; Xing, W.-R.; Zhou, Q.; Zheng, Y.-S.; Cao, H.-Y.; Zeng, C.-J. Surgical treatment for both-column acetabular fractures using pre-operative virtual simulation and three-dimensional printing techniques. Chin. Med. J. 2020, 133, 395-401. [CrossRef] [PubMed]

39. IJpma, F.F.A.; Meesters, A.M.L.; Merema, B.B.J.; Duis, K.; De Vries, J.P.M.; Banierink, H.; Wendt, K.W.; Kraeima, J.; Witjes, M.J.H. Feasibility of Imaging-Based 3-Dimensional Models to Design Patient-Specific Osteosynthesis Plates and Drilling Guides. JAMA Netw. Open 2021, 4, 1-13. [CrossRef] [PubMed]

40. Downey, C.; McCarrick, C.; Fenelon, C.; Murphy, E.P.; O’Daly, B.J.; Leonard, M. A novel approach using 3-D printing in the Irish National Centre for pelvic and acetabular surgery. Ir. J. Med. Sci. 2020, 189, 219-228. [CrossRef] [PubMed]

41. Hsu, C.-L.; Chou, Y.-C.; Li, Y.-T.; Chen, J.-E.; Hung, C.-C.; Wu, C.-C.; Shen, H.-C.; Yeh, T.-T. Pre-operative virtual simulation and three-dimensional printing techniques for the surgical management of acetabular fractures. Int. Orthop. 2019, 43, 1969-1976. [CrossRef] [PubMed]

42. Li, Y.T.; Hung, C.C.; Chou, Y.C.; Chen, J.E.; Wu, C.C.; Shen, H.C.; Yeh, T. Te Surgical treatment for posterior dislocation of hip combined with acetabular fractures using preoperative virtual simulation and three-dimensional printing model-assisted precontoured plate fixation techniques. Biomed Res. Int. 2019, 2019, 3971571. [CrossRef] [PubMed]

43. Maini, L.; Sharma, A.; Jha, S.; Sharma, A.; Tiwari, A. Three-dimensional printing and patient-specific pre-contoured plate: Future of acetabulum fracture fixation? Eur. J. Trauma Emerg. Surg. 2018, 44, 215-224. [CrossRef]

44. Maini, L.; Verma, T.; Sharma, A.; Sharma, A.; Mishra, A.; Jha, S. Evaluation of accuracy of virtual surgical planning for patient-specific pre-contoured plate in acetabular fracture fixation. Arch. Orthop. Trauma Surg. 2018, 138, 495-504. [CrossRef] [PubMed]

45. Öztürk, A.M.; Süer, O.; Şirintürk, S.; Aktuğlu, K.; Govsa, F.; Özer, M.A. A retrospective comparison of the conventional versus three-dimensional printed model-assisted surgery in the treatment of acetabular fractures. Acta Orthop. Traumatol. Turc. 2020, 54, 385-393. [CrossRef] [PubMed]

46. Wan, L.; Zhang, X.; Zhang, S.; Li, K.; Cao, P.; Li, J.; Wu, G. Clinical feasibility and application value of computer virtual reduction combined with 3D printing technique in complex acetabular fractures. Exp. Ther. Med. 2019, 3630-3636. [CrossRef] [PubMed]

47. Wang, C.; Chen, Y.; Wang, L.; Wang, D.; Gu, C.; Lin, X.; Liu, H.; Chen, J.; Wen, X.; Liu, Y.; et al. Three-dimensional printing of patient-specific plates for the treatment of acetabular fractures involving quadrilateral plate disruption. BMC Musculoskelet. Disord. 2020, 21, 1-9. [CrossRef]

48. Meesters, A.M.L.; Kraeima, J.; Banierink, H.; Slump, C.H.; de Vries, J.P.P.M.; ten Duis, K.; Witjes, M.J.H.; IJpma, F.F.A. Introduction of a three-dimensional computed tomography measurement method for acetabular fractures. PLoS ONE 2019, 14, e0218612. [CrossRef]

49. Verbeek, D.O.; van der List, J.P.; Villa, J.C.; Wellman, D.S.; Helfet, D.L. Postoperative CT is superior for acetabular fracture reduction assessment and reliably predicts hip survivorship. J. Bone Jt. Surg. 2017, 99, 1745-1752. [CrossRef]

50. Tannast, M.; Najibi, S.; Matta, J.M. Two to twenty-year survivorship of the hip in 810 patients with operatively treated acetabular fractures. J. Bone Jt. Surg. 2012, 94- A, 1559-1567. [CrossRef]

51. Banierink, H.; ten Duis, K.; Wendt, K.; Heineman, E.; IJpma, F.; Reininga, I. Patient-reported physical functioning and quality of life after pelvic ring injury: A systematic review of the literature. PLoS ONE 2020, 15, e0233226. [CrossRef]

52. Meesters, A.M.L.; ten Duis, K.; Banierink, H.; Stirler, V.M.A.; Wouters, P.C.R.; Kraeima, J.; de Vries, J.P.P.M.; Witjes, M.J.H.; IJpma, F.F.A. What Are the Interobserver and Intraobserver Variability of Gap and Stepoff Measurements in Acetabular Fractures? Clin. Orthop. Relat. Res. 2020, 478, 2801-2808. [CrossRef] 